\title{
Sarcoma de Ewing extraóseo
}

\author{
Extraosseous Ewing's sarcoma
}

\author{
José Hinojosa Gómez, ${ }^{\star}$ Alberto Villalobos Prieto, ${ }^{\ddagger}$ Raymundo Hernández Montes de Oca*
}

Citar como: Hinojosa GJ, Villalobos PA, Hernández MOR. Sarcoma de Ewing extraóseo. An Med ABC. $2021 ; 66$ (4): 283-287. https:// dx.doi.org/10.35366/102928

\section{RESUMEN}

Los tumores primarios de hueso son poco frecuentes, representan sólo 5\% de todos los tumores de los adolescentes y adultos jóvenes. De este grupo, el segundo lugar lo ocupa el sarcoma de Ewing óseo y extraóseo. Histológicamente se caracteriza por presentar células pequeñas, redondas y azules. Los síntomas se relacionan de manera directa con la localización y el tamaño del tumor. En general, el tratamiento se basa en quimioterapia seguida de terapia local (cirugía y/o radioterapia) y después quimioterapia. Caso clínico: Mujer de 70 años de edad que inició con una tumoración en la ingle derecha de crecimiento progresivo y acelerado. Se sospechó de una neoplasia hematológica y en el PET CT se detectó una masa de tejidos blandos en la región inguinal derecha de $45 \mathrm{~mm}$ con un valor estandarizado máximo de 9.8. Se realizó resección de la lesión y el estudio histopatológico reveló un ganglio linfático infiltrado por tumor neuroectodérmico primitivo/ sarcoma de Ewing. Después recibió radioterapia postoperatoria. El sarcoma de Ewing extraóseo es una enfermedad rara; en general, estos pacientes tienen mejor pronóstico que los pacientes con sarcoma de Ewing óseo. Al existir poca evidencia del tratamiento, éste debe de ser individualizado y por lo tanto, sometido a un abordaje multidisciplinario.

Palabras clave: Sarcoma, Ewing, extraóseo, radioterapia.

\section{INTRODUCCIÓN}

Los tumores primarios de hueso comprenden $5 \%$ de todos los tumores de los adolescentes y adultos jóvenes. Dentro de éstos, los segundos más comunes

\begin{abstract}
Primary bone tumors are rare, representing only $5 \%$ of all tumors in adolescents and young adults. Of this group, the second place is occupied by osseous and extraosseous Ewing's sarcoma. Histologically it is characterized by presenting small, round and blue cells. Symptoms are directly related to the location and size of the tumor. In general, treatment is based on chemotherapy followed by local therapy (surgery and/ or radiotherapy) and again chemotherapy. Clinical case: a 70-year-old woman was presented with a tumor in the right groin with progressive and accelerated growth. A hematological neoplasm was suspected and a $45 \mathrm{~mm}$ soft tissue mass in the right groin region with a standardized uptake value max of 9.8 was found on PET CT. A surgical resection was performed and in the histopathological study reported a lymph node infiltrated by a primitive neuroectodermal tumor/Ewing's sarcoma. After that, the patient was treated with postoperative radiotherapy. Extraosseous Ewing's sarcoma is a rare disease; in general, these patients have a better prognosis than patients with osseous Ewing's sarcoma. As there is little evidence of treatment, it must be individualized and therefore subjected to a multidisciplinary approach.
\end{abstract}

Keywords: Sarcoma, Ewing's, extraosseous, radiotherapy.

son los tumores de la familia del sarcoma de Ewing (TFSE). ${ }^{1}$ Los TFSE incluyen el sarcoma de Ewing (SE) óseo o extraóseo, tumor neuroectodérmico primitivo periférico, entre otros. Todos éstos comparten características genéticas e inmunohistoquímicas
* Radio-Oncología.
‡ Hemato-Oncología.

Centro Médico ABC. México.
Correspondencia:

Dr. Raymundo Hernández Montes de Oca

E-mail: dr.raymundo.hdez@gmail.com

Recibido: 26/05/2021. Aceptado: 20/09/2021. 
muy similares. ${ }^{1-3}$ Histológicamente, se caracterizan por presentar células pequeñas, redondas y azules. Son tumores pobremente diferenciados y altamente malignos sin diferenciación estructural. ${ }^{1-3}$

En México, de acuerdo a Rizo y colaboradores, ${ }^{4}$ los tumores de hueso y cartílago tienen una incidencia de $1.5 \%$ (hombres $0.9 \%$ y mujeres $0.6 \%$ ) y $21.9 \%$ de los casos se presentan en menores de 20 años de edad. En el periodo comprendido entre 2000 y 2004, sólo se reportaron nueve casos de SE. En Estados Unidos, la incidencia de SE óseo en el periodo entre 1973 y 2004 fue de 2.93 casos por cada millón de habitantes. La incidencia más alta se encuentra en la segunda década de la vida y llega a ser hasta de nueve a 10 casos por cada millón de habitantes; es más frecuente en hombres de raza caucásica, seguida de la asiática y afroamericana. Los pacientes con $\mathrm{SE}$ extraóseo por lo general son de mayor edad y es menos probable que se presente en hombres de raza blanca. ${ }^{5,6}$

La mayoría de los SE se ubican en el tejido óseo. Los sitios más afectados son los huesos planos del esqueleto axial (pelvis y caja torácica) y la diáfisis de los huesos largos de las extremidades inferiores (fémur), aunque se pueden presentar en cualquier hueso. De 16 a $25 \%$ de los tumores pueden tener origen extraóseo (tejidos blandos). ${ }^{7,8}$ Los sitios extraóseos más afectados son el espacio paravertebral, extremidades inferiores, cabeza y cuello, pelvis, retroperitoneo, omento, órbita, piel y pared torácica. ${ }^{9,10}$

Los síntomas se relacionan directamente con la localización y el tamaño del tumor, por lo tanto, se presentan como masa y/o dolor en el sitio del primario. En ocasiones pueden existir síntomas sistémicos tales como fiebre, pérdida de peso y fatiga. ${ }^{7-10}$

Los factores que indican un pronóstico favorable son: mujeres, localización primaria en extremidades (al contrario de pelvis), enfermedad localizada, tamaño $<8 \mathrm{~cm}$, volumen $<100 \mathrm{~mL}$, edad $<$ 15 años, deshidrogenasa láctica dentro de valores normales y buena respuesta a quimioterapia neoadyuvante. ${ }^{11}$

De acuerdo con las guías estadounidenses ${ }^{12}$ y europeas, ${ }^{13}$ el tratamiento del SE óseo o extraóseo es con $\mathrm{VAC} / \mathrm{IE}^{14,15}$ (vincristina, doxorrubicina y ciclofosfamida alternando con ifosfamida y etopósido) por al menos nueve semanas (de tres a seis ciclos), terapia local (cirugía $[\mathrm{CX}]$ y/o radioterapia $[\mathrm{RT}]$ ) y posteriormente de nuevo quimioterapia (QT) por seis a 10 ciclos, por lo regular aplicados cada dos a tres semanas. La duración aproximada del tratamiento es de 10 a 12 meses. Siempre que el tumor sea completamente resecable debe ser sometido a $\mathrm{CX}$, de lo contrario se utilizará RT definitiva con dosis de 45 Gy a 60 Gy, dependiendo de la localización del tumor y de las restricciones de los órganos de riesgo. La RT postoperatoria a dosis de 45 Gy a 60 Gy está indicada en caso de márgenes inadecuados (positivos o cercanos) y se discute en pacientes con respuesta parcial a QT. En pacientes con SE extraóseo, la RT postoperatoria se indica en todos los pacientes, excepto en aquéllos con buen pronóstico o SE superficial. ${ }^{12,13}$

Debido a la escasa información de calidad existente, revisaremos el papel de la RT en el sarcoma de Ewing enfocándonos en la enfermedad extraósea.

\section{CASO CLÍNICO}

Mujer de 70 años de edad sin antecedentes médicos de importancia. Un mes previo al diagnóstico detectó una tumoración en la ingle derecha de crecimiento progresivo y acelerado. Acudió con un médico, quien ante los hallazgos sospechó de un tumor maligno y solicitó PET CT, el cual mostró una imagen sugerente de masa de tejidos blandos en la región inguinal derecha de $45 \mathrm{~mm}$ en su eje corto que refuerza con la aplicación de medio de contraste, alternando con imágenes hipodensas en su interior, con adecuada interfase con los músculos adyacentes, la cual tiene SUV máximo de 9.8 y en la adquisición tardía SUV máximo de 10.6. Fue sometida a resección de la lesión y el estudio histopatológico reveló linfangioleimiomatosis con tumor neuroectodérmico primitivo (sarcoma de Ewing), CD99 positivo, con bajo índice de proliferación. Márgenes quirúrgicos negativos. Se realizó una segunda revisión por parte de patología y se corroboró lo anterior, ganglio linfático infiltrado por tumor neuroectodérmico primitivo/sarcoma de Ewing. Después fue enviada para valorar RT postoperatoria. De acuerdo con la información existente se consideró candidata al tratamiento, por lo que brindamos RT conformada al sitio original del tumor (previa fusión con PET CT prequirúrgico) con márgenes para CTV de $1.5 \mathrm{~cm}$ y para PTV de $0.5 \mathrm{~cm}$. La dosis prescrita fue de 50.4 Gy en 28 fracciones (1.8 Gy/fracción) (Figura 1). Durante la administración de la radioterapia no hubo toxicidad relacionada con el tratamiento. Actualmente la paciente tiene una supervivencia libre de enfermedad de 45 meses y no hay toxicidad debido al tratamiento. En el último PET CT de control no hay datos de recurrencia de la enfermedad (Figura 2). 


\section{DISCUSIÓN}

El SE es un tumor radiosensible y la enfermedad localizada puede tener una excelente respuesta con $\mathrm{RT}$ como modalidad única de tratamiento. La CX se considera el tratamiento local de elección para este grupo de pacientes, ya que en la mayoría de los estudios los pacientes tienen mejor control local (CL) que aquellos que recibieron únicamente RT. En el estudio realizado por Schuck ${ }^{16}$ se analizó el impacto de la terapia local en pacientes con enfermedad localizada. El tratamiento consistió en una combinación de QT y terapia local (CX con/sin RT postoperatoria, RT sola o RT preoperatoria). La falla local (FL) a cinco años fue de $7.5 \%$ después de CX con/sin RT postoperatoria, de $5.3 \%$ en RT preoperatoria y de $26.3 \%$ después de $R T$ definitiva $(p=0.001)$. La supervivencia libre de evento (SLE) a 10 años en pacientes que recibieron RT definitiva fue de $40 \%$, RT preoperatoria de $58 \%$ y CX con/sin RT de 55\%. ${ }^{17-20}$ Hasta el momento no existen estudios aleatorizados que comparen directamente CX contra RT.

En los estudios CESS $^{16-18}$ y EICESS ${ }^{16,19,20}$ los pacientes con márgenes cercanos y/o pobre respuesta a la QT recibieron RT postoperatoria. Los pacientes con resecciones marginales con/sin RT tuvieron una FL de 6\%, sin embargo, se observó que los pacientes que recibieron $\mathrm{RT}$ tuvieron menor respuesta a la QT, comparada con aquellos que no la recibieron (39\% versus $72 \%$ ). Los pacientes con resección amplia y pobre respuesta a QT que no recibieron RT tuvieron una FL de $12 \%$ y los que sí la recibieron de 6\%. Los pacientes con resección amplia y buena respuesta a QT con/sin RT en la FL fueron de 1 y $6 \%$, respectivamente. Por lo tanto, de acuerdo a la poca evidencia existente y a pesar de los sesgos en la selección, los pacientes con márgenes positivos o cercanos ${ }^{21}(<1$ $\mathrm{cm}$ para hueso, $<5 \mathrm{~mm}$ para grasa y músculo $\mathrm{y}<2$ $\mathrm{mm}$ para fascias) y/o pobre respuesta a $\mathrm{QT}^{16,19,20}(<$ $50 \%$ ) se benefician de RT postoperatoria.

Las indicaciones de RT preoperatoria se basan en el estudio de Schuck, ${ }^{16}$ ya que el CL en este subgrupo fue excelente, con una tasa de FL y sistémica combinada de $6 \%$ a cinco años. De acuerdo al estudio, los pacientes que se benefician de RT preoperatoria son los que se consideran marginalmente resecables, inoperables y/o que tienen una respuesta inferior a 50\% después del segundo ciclo de QT. Los beneficios de la RT preoperatoria en sarcomas de tejidos blandos son: facilitan la resección, disminuyen el riesgo de siembra microscópica, volumen de tratamiento bien definido y por lo tanto, menor cantidad de tejido sano irradiado y flujo sanguíneo adecuado al tumor que en teoría puede mejorar la respuesta al tratamiento. El riesgo de administrar radioterapia preoperatoria es que aumentan las complicaciones relacionadas con la herida. ${ }^{22}$

Las lesiones que se consideran no resecables con márgenes amplios o donde la CX es muy mutilante (columna vertebral, base de cráneo, cabeza y cuello) deben recibir RT definitiva como única forma de tratamiento local. ${ }^{12,13,16}$ Cuando se utiliza RT defi-

Figura 1:

Plan de tratamiento de radioterapia conformada. Las líneas amarillas representan la curva de dosis de prescripción. Cortes de axial, sagital y coronal.

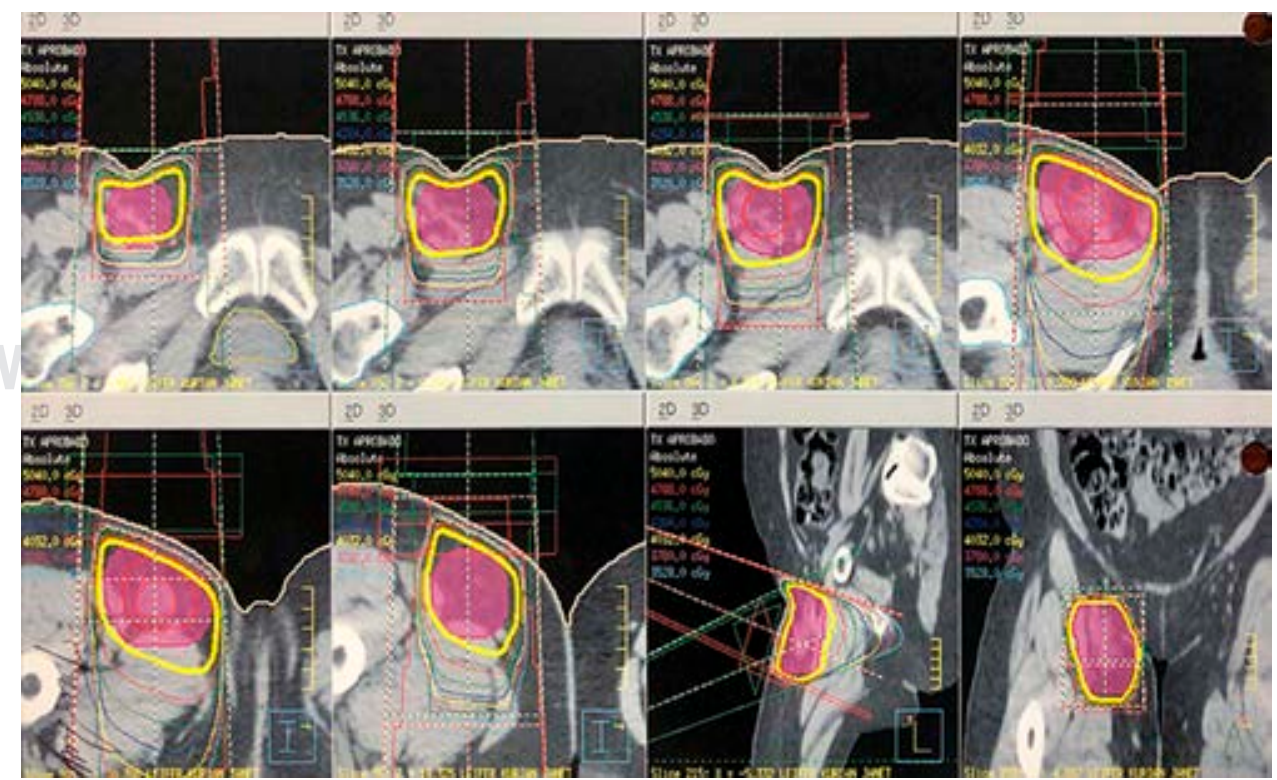




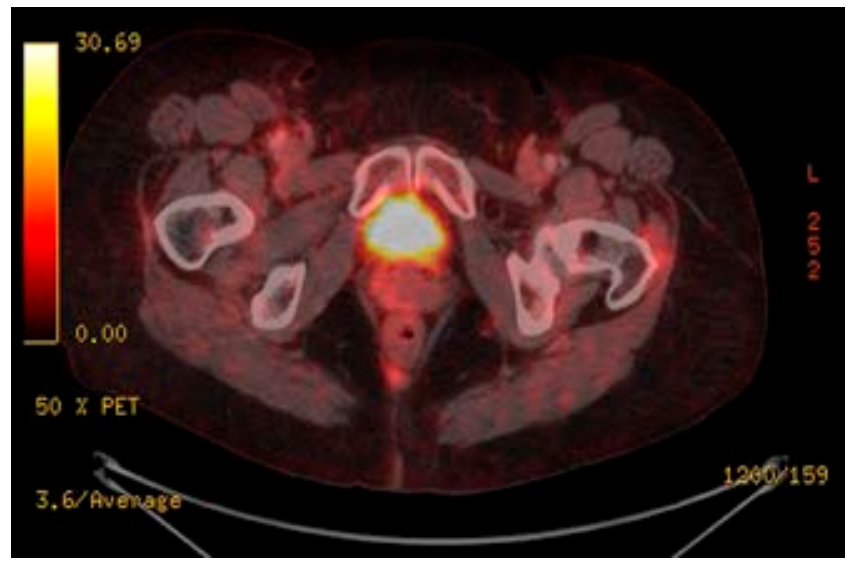

Figura 2: Corte axial de PET CT sin datos anatómicos o metabólicos de actividad tumoral.

nitiva, el CL es inferior en comparación con los pacientes que son tratados con CX con/sin RT. La recaída local y sistémica es de $26.3 \%$ en pacientes que sólo reciben RT, de $5.3 \%$ después de $\mathrm{RT}$ preoperatoria y de $7.5 \%$ en pacientes luego de $\mathrm{CX}$ con/sin $\mathrm{RT}$. Lo mismo sucede con la SLE, la cual a cinco años es de 47,59 y $61 \%$, respectivamente (p 0.0001). ${ }^{16}$ Es importante mencionar que estos pacientes son los que tienen el mayor número de factores pronósticos desfavorables tales como tumores de localización central, tamaño $>8 \mathrm{~cm}$, volumen $>100 \mathrm{~cm}^{3}$ y mala respuesta a QT. ${ }^{11,16,21}$

El SE extraóseo constituye $16 \%$ de todos los $\mathrm{SE}{ }^{23} \mathrm{Al}$ comparar el SE extraóseo y el óseo se encontró que los primeros tienen mejor SLE (76 versus $69 \%$ p 0.05 ) y SG (85 versus $78 \%$ p 0.11 ) a cinco años. ${ }^{24} \mathrm{Al}$ tener una incidencia muy baja, los esquemas de QT y RT se extrapolan del SE óseo. ${ }^{21,23,25,26}$ Las indicaciones de RT son las mismas, excepto en el caso de RT postoperatoria, en la que se puede evitar en pacientes con buen pronóstico y enfermedad superficial. ${ }^{13}$ Los pacientes con SE extraóseo con enfermedad subcutánea tienen una SLE a 10 años de $89.9 \%$ en comparación con $56.4 \%$ con enfermedad no subcutánea y $47.6 \%$ para enfermedad ósea. ${ }^{21,23,25-27}$

La dosis de RT para el SE óseo y extraóseo depende de la localización del tumor, secuencia y finalidad del tratamiento. La RT definitiva se administra a dosis de 45-60 Gy. La dosis de RT preoperatoria es de 36-45 Gy. En el caso de RT postoperatoria para márgenes negativos se recomienda una dosis de $45 \mathrm{~Gy}$, para márgenes con enfermedad microscópica 45-50.4 Gy y en caso de márgenes con enfermedad residual macroscópica la dosis será de 45-60 Gy. ${ }^{12,13,16-21,27,28}$

\section{CONCLUSIONES}

El sarcoma de Ewing extraóseo es una enfermedad rara; en general, estos pacientes tienen mejor pronóstico que los pacientes con sarcoma de Ewing óseo. $\mathrm{Al}$ existir poca evidencia del tratamiento, éste debe de ser sometido a una discusión multidisciplinaria y por lo tanto, debe ser individualizado.

\section{REFERENCIAS}

1. Balamuth NJ, Womer RB. Ewing's sarcoma. Lancet Oncol. 2010; 11 (2): 184-192.

2. Rekhi B, Vogel U, Basak R, Desai SB, Jambhekar NA. Clinopathological and molecular Spectrum of Ewing Sarcomas/PNET's, including validation of EWSR1 rearrangement by conventional and array FISH technique in certain cases. Pathol Oncol Res. 2014; 20 (3): 503-516.

3. Choi EY, Gardner JM, Lucas DR, McHugh JB, Patel RM. Ewing sarcoma. Semin Diagn Pathol. 2014; 31 (1): 39-47.

4. Rizo P, Sierra M, Vázquez G, Cano M, Meneses A, Mohar A. Registro hospitalario de cáncer: compendio de cáncer 20002004. Cancerología. 2007; 2: 203-287.

5. Esiashvili N, Goodman M, Marcus RB Jr. Changes in incidence and survival of Ewing sarcoma patients over the past 3 decades: surveillance epidemiology and end results data. J Pediatr Hematol Oncol. 2008; 30 (6): 425-430.

6. Jawad MU, Cheung MC, Min ES, Schneiderbauer MM, Koniaris LG, Scully SP. Ewing sarcoma demonstrates racial disparities in incidence-related and sex-related differences in outcome: an analysis of 1,631 cases from the SEER database, 1973-2005. Cancer. 2009; 115 (15): 3526-3536.

7. Bernstein M, Kovar H, Paulussen M, Randall RL, Schuck A, Teot LA et al. Ewing's sarcoma family of tumors: current management. Oncologist. 2006; 11 (5): 503-519.

8. Applebaum MA, Worch J, Matthay KK, Goldsby R, Neuhaus J, West DC, Dubois SG. Clinical features and outcomes in patients with extraskeletal Ewing sarcoma. Cancer. 2011; 117 (13): 3027-3032.

9. Cheung CC, Kandel RA, Bell RS, Methews RE, Ghazarian MD. Extraskeletal Ewing sarcoma in a 77-years-old woman. Arch Pathol Lab Med. 2001; 125 (10): 1358-1360.

10. Geens L, Van Robays J, Geert V, Van der Speeten K. An unusual location of extraosseous Ewing's sarcoma. Case Rep Oncol. 2013; 6 (2): 293-302.

11. Leavey PJ, Collier AB. Ewing sarcoma: prognostic criteria, outcomes and future treatment. Expert Rev Anticancer Ther. 2008; 8 (4): 617-624.

12. NCCN Guidelines Version 1.2020 Bone Cancer.

13. Bone sarcomas: ESMO-PaedCan-EURACAN Clinical Practice Guidelines for diagnosis, treatment and follow-up. Ann Oncol. 2018; 29 (Suppl 4): iv79-iv95.

14. Grier HE, Krallo MD, Tarbell NJ, Link MP, Fryer CJ, Pritchard DJ et al. Addition of ifosfamide and etoposide to standard chemotherapy for Ewing's sarcoma and primitive neuroectodermal tumor of bone. N Engl J Med. 2003; 348 (8): 694-701.

15. Kolb EA, Kushner BH, Gorlick R, Lavardiere C, Healey JH, LaQuaglia MP et al. Long-term event-free survival after 
An Med ABC. 2021; 66 (4): 283-287

intensive chemotherapy for Ewing's family of tumors in children and young adults. J Clin Oncol. 2003; 21 (18): 3423-3430.

16. Schuck A, Ahrens S, Paulussen M, Kuhlen M, Konemann $\mathrm{S}$, Rübe $\mathrm{C}$ et al. Local therapy in localized Ewing tumors: results of 1058 patients treated in the CESS 81, CESS 86, and EICESS 92 trials. Int J Radiat Oncol Biol Phys. 2003; 55 (1): 168-177.

17. Jurgens H, Gobel V, Michaelis J, Ramach W, Ritter J, Sauer R. The Cooperative Ewing Sarcoma Study CESS 81 of the German Pediatric Oncology Society analysis after 4 years. Klin Padiatr. 1985; 197 (3): 225-232.

18. Dunst J, Jurgens H, Sauer R, Pape H, Paulussen M, Winkelmann W, Rübe C. Radiation therapy in Ewing's sarcoma: an update of the CESS 86 trial. Int J Radiat Oncol Biol Phys. 1995; 32 (4): 919-930.

19. Paulussen M, Ahrens S, Braun-Munzinger G, Craft AW, Dockhorn-Dworniczak, Dorffel W et al. EICESS 92 (European Intergroup Cooperative Ewing's Sarcoma Study) - preliminary results. Klin Padiatr. 1999; 211 (4): 276-283.

20. Paulussen M, Craft AW, Lewis I, Hackshaw A, Douglas C, Dunst J. Results of the EICESS-92 Study: two randomized trials of Ewing's sarcoma treatment-cyclophosphamide compared with ifosfamide in standard risk patients and assessment of benefit of etoposide added to standard treatment in high-risk patients. J Clin Oncol. 2008; 26 (27): 4385-4393.

21. Granowetter L, Womer R, Devidas M, Krailo M, Wang C, Bernstein M. Dose-intensified compared with standard chemotherapy for nonmetastatic Ewing sarcoma family of tumors: a Children's Oncology Group Study. J Clin Oncol. 2009; 27 (15): 2536-2541.
22. O'Sullivan B, Davis AM, Turcotte R, Bell R, Catton C, Chabot P. Preoperative versus postoperative radiotherapy in softtissue sarcoma of the limbs: a randomised trial. Lancet. 2002; 359 (9325): 2235-2241.

23. Biswas B, Shukla NK, Deo S, Agarwala S, Sharma D, Vishnubhatla S et al. Evaluation of outcome and prognostic factors in extraosseous Ewing sarcoma. Pediatr Blood Cancer. 2014; 61: 1925-1931.

24. Cash T, McIlvaine E, Krailo MD, Lessnick SL, Lawlor ER, Laack $\mathrm{N}$ et al. Comparison of clinical features and outcomes in patients with extraskeletal versus skeletal localized Ewing sarcoma: a report from the Children's Oncology Group. Pediatr Blood Cancer. 2016; 63 (10): 1771-1779. doi: 10.1002/ pbc.26096.2016 14.

25. Orr WS, Denbo JW, Billups CA, Wu J, Navid F, Rao BN, Davidoff AM, Krasin MJ. Analysis of prognostic factors in extraosseous Ewing sarcoma family of tumors: review of St. Jude Children's Research Hospital experience. Ann Surg Oncol. 2012; 19 (12): 3816-3822.

26. Raney R, Asmar L, Newton W, Bagwell C, Breneman J, Gehan E et al. Ewing's sarcoma of soft tissues in childhood: a report from the Intergroup Rhabdomyosarcoma Study, 1972 to 1991. J Clin Oncol. 1997; 15 (2): 574-582.

27. Castex MP, Rubie H, Stevens M, Escribano C, Gauzy J, Brouchet A et al. Extraosseus localized Ewing tumors: improved outcome with anthracyclines -The French Society of Pediatric Oncology and International Society of Pediatric Oncology. J Clin Oncol. 2007; 25: 1176-1182.

28. Donaldson SS. Ewing sarcoma: radiation dose and target volumen. Pediatr Blood Cancer. 2004; 42 (5): 471-476. 